\title{
DISTORTION CANCELLATION VIA POLYPHASE MULTIPATH CIRCUITS
}

\author{
Eisse Mensink, Eric A. M. Klumperink, Bram Nauta \\ University of Twente, Enschede, The Netherlands \\ Email: e.mensink@utwente.nl
}

\begin{abstract}
The central question of this paper is: can we enhance the spectral purity of nonlinear circuits with the help of polyphase multipath circuits. Polyphase multipath circuits are circuits with two or more paths that exploit phase differences between the paths to cancel unwanted signals. It turns out that it is very well possible to cancel distortion products produced by a nonlinear circuit. Unfortunately, there are also some spectral components that cannot be canceled with the poylyphase multipath circuits. In this paper tables are presented that can easily be used to predict which spectral components are canceled and which are not canceled for a certain poylyphase multipath circuit.
\end{abstract}

\section{INTRODUCTION}

Transistor circuits can introduce unwanted spectral components due to the nonlinear component characteristics [1]. Linearization by feedback is possible but with limits. Especially in high frequency circuits, the amount of available loop gain is limited. Furthermore, feedback introduces instability risks.

Another commonly used method of removing unwanted spectral components is filtering. However, the achievable Q is limited, impeding steep filtering. Moreover, the required filters are application specific, which is for instance a problem in realizing multi-standard radio transceiver systems.

Thus alternative more flexible techniques for spectral purity enhancement are very much wanted. This paper explores the possibility of polyphase multipath circuits to reject unwanted spectral components. Polyphase multipath circuits are circuits with two or more paths that exploit phase differences between the paths to cancel unwanted signals. Examples with two paths are balanced circuits and circuits for image rejection [2]. This paper takes a generalized look at circuits with two or more paths.

The key aim is to find out to what extend polyphase multipath circuits can cancel distortion. It turns out that most of the unwanted spectral components can be canceled if a sufficient number of paths and phases is used, but there are also some components that cannot be canceled.

The contents of the paper are structured as follows. Section 2 describes and analyses the polyphase multipath technique. In section 3, the results of the analysis of the polyphase multipath technique are compared with some known techniques that cancel unwanted spectral components. Section 4 gives simulation

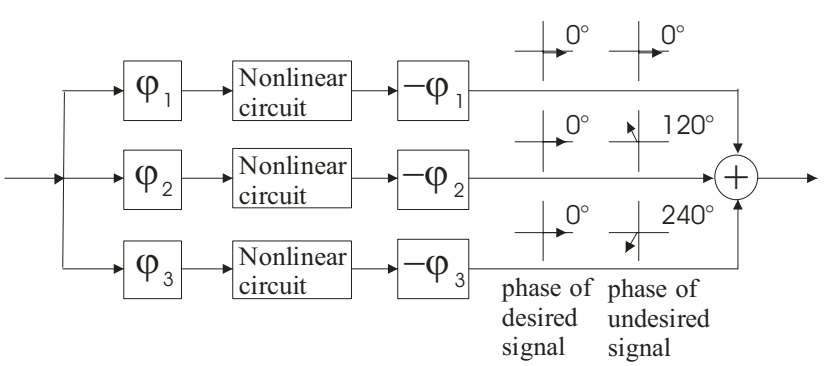

Figure 1: Polyphase multipath circuit with $N=3$ paths.

results that support the analysis of section 2 and section 5 gives the conclusions.

\section{POLYPHASE MULTIPATH TECHNIQUE}

\subsection{Distortion products}

Unwanted spectral components often result from nonlinearity, e.g. in RF amplifiers, filters and mixers. A good overview of distortion mechanisms and analysis is given in a paper by Sansen [1].

If $x(t)=A_{1} \cos \left(\omega_{1} t\right)+A_{2} \cos \left(\omega_{2} t\right)$ is the input of a nonlinear circuit, the output will not only consist of cosines with a fundamental frequency $\left(\omega_{1}\right.$ or $\left.\omega_{2}\right)$, but also of cosines with other frequencies: harmonics $\left(\mathrm{n} \omega_{1}\right.$ or $\left.\mathrm{n} \omega_{2}\right)$ and intermodulation products $\left(\mathrm{k} \omega_{1}+\mathrm{m} \omega_{2}\right)$.

The question that we address in this paper is: can we cancel the harmonics and intermodulation products with the help of a polyphase multipath technique?

\subsection{Polyphase multipath technique}

Figure 1 shows a polyphase multipath circuit with three paths ( $\mathrm{N}$ $=3$ ). The basic idea is to split an input signal into $\mathrm{N}$ paths that consist of a phase shifter, a nonlinear circuit with distortion to be canceled, and another phase shifter. The nonlinear circuits are identical, but the phase shifters have a different phase in every path. At the end, the outputs of the different paths are added to get the output signal.

The aim of the circuit of figure 1 is twofold. On the one hand, the desired signals should have equal phases at the end of every path. In this way they add constructively. On the other hand, the undesired signals (distortion products) should have a different phase at the end of every path and the phase differences between the paths should be chosen in such a way that the 


\begin{tabular}{|l|c|c|c|c|c|c|c|c|}
\hline $\mathbf{n}^{\text {th }}$ harmonic & $\mathbf{1}$ & $\mathbf{2}$ & $\mathbf{3}$ & $\mathbf{4}$ & $\mathbf{5}$ & $\mathbf{6}$ & $\mathbf{7}$ & $\mathbf{8}$ \\
\hline phase at output & $\underline{0}$ & $\underline{\varphi}$ & $2 \underline{\varphi}$ & $3 \underline{\varphi}$ & $4 \underline{\varphi}$ & $5 \underline{\varphi}$ & $6 \underline{\varphi}$ & $7 \underline{\varphi}$ \\
\hline
\end{tabular}

Table 1: The phase of the $\mathrm{n}^{\text {th }}$ harmonic just before the addition in a polyphase multipath circuit.

\begin{tabular}{|c|c|c|c|c|c|c|c|c|}
\hline $\begin{array}{l}\text { phase at output } \\
\text { \# of paths (N) }\end{array}$ & $\underline{\mathbf{0}}$ & $\pm \varphi$ & $\pm 2 \varphi$ & $\pm 3 \varphi$ & $\pm 4 \varphi$ & $\pm 5 \varphi$ & $\pm 6 \varphi$ & $\pm 7 \varphi$ \\
\hline 2 & & $\mathrm{C}$ & & $\mathrm{C}$ & & $\mathrm{C}$ & & $\mathrm{C}$ \\
\hline 3 & & $\mathrm{C}$ & $\mathrm{C}$ & & $\mathrm{C}$ & $\mathrm{C}$ & & $\mathrm{C}$ \\
\hline 4 & & $\mathrm{C}$ & $\mathrm{C}$ & $\mathrm{C}$ & & $\mathrm{C}$ & $\mathrm{C}$ & $\mathrm{C}$ \\
\hline 5 & & $\mathrm{C}$ & $\mathrm{C}$ & $\mathrm{C}$ & $\mathrm{C}$ & & $\mathrm{C}$ & $\mathrm{C}$ \\
\hline
\end{tabular}

Table 2: This table indicates whether harmonics are canceled ("C") or not (blank) depending on their phase (columns) and number of paths (rows).

unwanted signals are canceled.

How can this situation be achieved? We can find this out by looking at the phases of the signals in the different paths. First, we will analyze the harmonics of the input signal, where the first harmonic is the desired signal. Intermodulation products will be looked at afterwards.

\subsection{Canceling of harmonics}

If we combine the phases of the phase shifters in the different paths into a vector $\varphi=\left[\varphi_{1}, \varphi_{2}, \ldots, \varphi_{N}\right]$, then the phases of the signals after the first phase shifters are $\varphi$ (assuming $\varphi_{\text {in }}=0$ ). The nonlinear circuits generate harmonics of these signals. The phases of the $\mathrm{n}^{\text {th }}$ harmonic after the nonlinear circuits will be

$$
\underline{\varphi}_{o u t_{-} n l}=n \cdot \underline{\varphi}
$$

This relation results from the fact that in a (weakly) nonlinear circuit the phase of a cosine undergoes the same operations as the frequency of the cosine: $\cos ^{\mathrm{n}}\left(\omega^{*} \mathrm{t}+\varphi_{\mathrm{i}}\right)$ leads to $\cos \left(\mathrm{n} * \omega * \mathrm{t}+\mathrm{n} * \varphi_{\mathrm{i}}\right)$.

The phase shifters after the nonlinear circuits set the phases of the desired signals $(n=1)$ back to zero. The higher harmonics undergo the same phase shifts (the phase shifters are assumed to be frequency independent). Therefore, the phases of the $\mathrm{n}^{\text {th }}$ harmonic just before the adder will be

$\underline{\varphi}_{\text {out }}=(n-1) \cdot \underline{\varphi}$

The desired signals will have the same phase in every path $\left(\underline{\varphi}_{\text {out }}=\underline{0}\right)$ and add constructively. The higher harmonics will have a different phase in every path $\left(\underline{\varphi}_{\text {out }} \neq \underline{0}\right)$ and can be canceled. It depends on the choice of $\varphi$ and the number of paths, $\mathrm{N}$, whether these harmonics are canceled or not.

Table 1 and 2 can be used to find out which harmonics are canceled for a certain number of paths. First, table 1 has to be used to find the phase of a harmonic (table 1 is produced with the help of equation 2). Then table 2 can be used to see if this phase is canceled by a certain number of paths. Table 2 is valid for the case that the phases between the paths are chosen equidistant, thus $\varphi=\left[0^{*} 360^{\circ} / \mathrm{N}, 1^{*} 360^{\circ} / \mathrm{N}, \ldots,(\mathrm{N}-1)^{*} 360^{\circ} / \mathrm{N}\right]$.

Table 1 and 2 show that the more paths are used, the more harmonics can be canceled. For $\mathrm{N}$ paths, the $\left(\mathrm{p}^{*} \mathrm{~N}+1\right)^{\text {th }}$ harmonics $(p=0,1,2, \ldots)$ are the only harmonics that are not canceled. The reason for this can easily be seen with the help of equation 2 . With $\mathrm{n}=\mathrm{p} * \mathrm{~N}+1$ equation 2 becomes $\varphi_{\text {out }}=\mathrm{p}^{*} \mathrm{~N}^{*} \varphi=$ $\mathrm{p} *\left[0^{*} 360^{\circ}, 1 * 360^{\circ}, \ldots,(\mathrm{N}-1)^{*} 360^{\circ}\right]$, which means the same phase in every path, i.e. constructive addition. In all other cases, the phases are distributed equidistantly over $360^{\circ}$, leading to cancellation.

\subsection{Canceling of intermodulation products}

The canceling of intermodulation products can be analyzed in the same way as the canceling of the harmonics. Similar to equations 1 and 2, it can be shown that intermodulation products at $\mathrm{k} \omega_{1}+\mathrm{m} \omega_{2}$ ( $\mathrm{k}$ and $\mathrm{m}$ can be positive and negative) will have a phase of $(\mathrm{k}+\mathrm{m})^{*} \varphi$ after the nonlinear circuits and a phase of $(\mathrm{k}+\mathrm{m}-1)^{*} \underline{\varphi}$ before the adder. The only difference with equation 2 is that $\mathrm{n}$ is replaced by $\mathrm{k}+\mathrm{m}$. Therefore, if the $\mathrm{n}^{\text {th }}$ harmonic is canceled, also the intermodulation products at $\mathrm{k} \omega_{1}+\mathrm{m} \omega_{2}$ with $\mathrm{k}+\mathrm{m}=\mathrm{n}$ will be canceled.

Comparable to the results of section 2.3 , the only intermodulation products that are not canceled are the intermodulation products with $\mathrm{k}+\mathrm{m}=\mathrm{p} * \mathrm{~N}+1$. Unfortunately, this means that the intermodulation products that have $\mathrm{k}+\mathrm{m}=1$ can never be canceled, because otherwise the desired signals ( $\mathrm{n}$ $=1$ ) would also be canceled. The most important intermodulation products that are in this category are third order intermodulation products at $2 \omega_{1}-\omega_{2}$ and $2 \omega_{2}-\omega_{1}$. Unfortunately, these products are often a big problem in RF receivers.

Because the intermodulation products with $\mathrm{k}+\mathrm{m}=\mathrm{n}$ will be canceled whenever the $\mathrm{n}^{\text {th }}$ harmonic is canceled, table 1 and 2 can also be used for intermodulation products: just replace $n$ by $\mathrm{k}+\mathrm{m}$. In the remainder of this paper, for briefness we will only discuss the canceling of harmonics.

\subsection{Mixer as a wideband phase shifter}

The phase shifter after the nonlinear circuit has to perform the same phase shift on every harmonic or at least on those harmonics that are in the frequency band of interest. If it is not possible to make such a wideband phase shifter with passive RLC filter networks, a mixer can be used [3]. A mixer as a phase shifter will be very wideband. Figure 2 shows the system with mixers. The mixers in this figure are assumed to be ideal mixers (mixer nonlinearity is modeled in the nonlinear circuits).

However, the mixer will introduce not only a phase shift, but also a frequency shift. So this technique can only be used if 


\begin{tabular}{|c|c|c|c|c|c|c|c|c|c|c|c|}
\hline $\begin{array}{l}\mathrm{n}^{\text {th }} \text { harmonic (input) } \\
\mathrm{j}^{\text {th }} \text { harmonic (LO) }\end{array}$ & -5 & -4 & -3 & -2 & -1 & 0 & 1 & 2 & 3 & 4 & 5 \\
\hline $\mathbf{0}$ & $5 \varphi$ & $4 \varphi$ & $3 \varphi$ & $2 \varphi$ & $\varphi$ & $\underline{0}$ & $\varphi$ & $2 \varphi$ & $3 \varphi$ & $4 \varphi$ & $5 \varphi$ \\
\hline 1 & $6 \varphi$ & $5 \varphi$ & $4 \varphi$ & $3 \varphi$ & $2 \varphi$ & $-\varphi$ & $\underline{0}$ & $\varphi$ & $2 \varphi$ & $3 \varphi$ & $4 \varphi$ \\
\hline 2 & $7 \underline{\varphi}$ & $6 \underline{\varphi}$ & $5 \underline{\varphi}$ & $4 \underline{\varphi}$ & $3 \underline{\varphi}$ & $-2 \underline{\varphi}$ & $-\varphi$ & $\underline{0}$ & $\underline{\varphi}$ & $2 \underline{\varphi}$ & $3 \underline{\varphi}$ \\
\hline 3 & $8 \underline{\varphi}$ & $7 \varphi$ & $6 \varphi$ & $5 \varphi$ & $4 \varphi$ & $-3 \varphi$ & $-2 \varphi$ & $-\varphi$ & $\underline{0}$ & $\varphi$ & $2 \varphi$ \\
\hline 4 & $9 \underline{\varphi}$ & $8 \underline{\varphi}$ & $7 \underline{\varphi}$ & $6 \underline{\varphi}$ & $5 \underline{\varphi}$ & $-4 \varphi$ & $-3 \underline{\varphi}$ & $-2 \varphi$ & $-\varphi$ & $\underline{0}$ & $\underline{\varphi}$ \\
\hline
\end{tabular}

Table 3: The phase of the $n^{\text {th }}$ harmonic mixed with the $j^{\text {th }}$ harmonic of the LO signal just before the addition in a polyphase multipath circuit if a mixer is used.

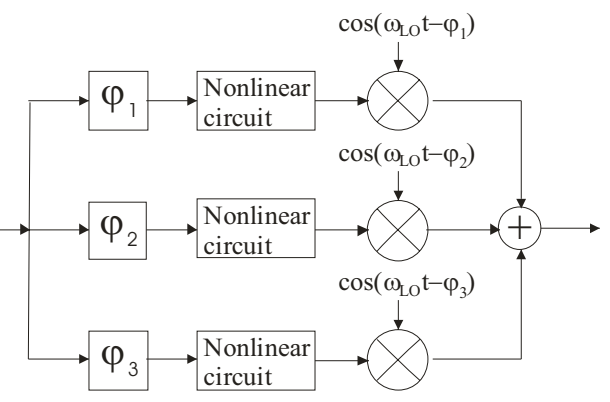

Figure 2: Mixers instead of phase shifters $(\mathbf{N}=3)$.

this frequency shift is desired (e.g. in an upconverter).

Furthermore, extra spectral components are introduced: due to sum and difference products of the input signal with the local oscillator (LO) signal, both an upper and a lower sideband will appear. So in the frequency spectrum, harmonics will appear at both sides of the LO frequency.

Another problem that can appear in the mixer, is the presence of harmonics of the LO signal (for instance in a switching mixer). These harmonics of the LO signal will also be mixed with the input signal of the mixer and give even more extra spectral components. In the frequency spectrum, the harmonics of the input signal will also appear at both sides of harmonics of the LO signal.

Assume now, that the desired signal is in the upper sideband, while a positive $\mathrm{n}$ means that the harmonic is in the upper sideband and a negative $n$ means that the harmonic is in the lower sideband. ${ }^{1}$ The formula for the phases at the outputs of the mixers for the $\mathrm{n}^{\text {th }}$ harmonic of the input signal and the $\mathrm{j}^{\text {th }}$ harmonic of the LO signal is:

$\underline{\varphi}_{\text {out }}=\left\{\begin{array}{rr}(n-j) \cdot \underline{\varphi} & n \geq 0 \\ (-n+j) \cdot \underline{\varphi} & n<0\end{array}\right.$

Table 3 shows the result of equation 3 for common values of $\mathrm{n}$ and $\mathrm{j}$. Table 3 can be used in the same way as table 1: the phases of a certain spectral component can be found in this table. Then, table 2 can be used to see how many paths are needed to cancel that component. It turns out that most of the spectral

${ }^{1}$ If the desired signal is in the lower sideband, the phases of the LO signals in figure 2 should be made positive instead of negative. Then, a positive $\mathrm{n}$ means lower sideband and a negative $\mathrm{n}$ means upper sideband.

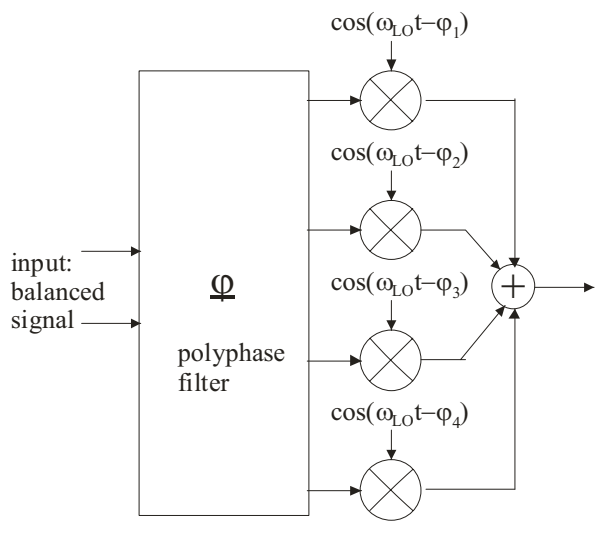

Figure 3: The image reject structure of Gingell $(N=4)$.

components can be canceled if the right number of paths is chosen. The only spectral components that the system of figure 2 does not cancel are the spectral components with $\mathrm{n}=\mathrm{j}+\mathrm{p}^{*} \mathrm{~N}(\mathrm{p}=$ $\ldots,-2,-1,0,1,2, \ldots)$.

Unfortunately, table 3 shows that components with $n=j(p$ $=0$ ) always have $\underline{\varphi}_{\text {out }}=\underline{0}$, independently of $\mathrm{N}$. This means that they cannot be canceled with any number of paths.

\section{COMPARISON WITH OTHER TECHNIQUES}

In this section, two known techniques are analyzed using table 1 to 3. It is shown that both techniques can be explained with the help of these tables. However, it is also made clear that the tables are more general. First, balanced (or differential) circuits are examined. After that, the image reject structure as proposed by Gingell [2] is considered.

Balanced circuits are known for their ability to cancel all the even harmonics of a nonlinear circuit. The nonlinear circuit is copied to create two paths. The inputs of the two paths are driven in antiphase and the output is the difference between the outputs of the two paths. Comparing this with table 1 and 2 , balanced circuits can be considered as polyphase multipath circuits with $\mathrm{N}=2, \varphi=\left[0^{\circ}, 180^{\circ}\right]$. The phase shifts of figure 1 are performed with minus signs, which are very wideband phase shifters (recall that wideband phase shifters were needed to make the technique work). However, while balanced circuits cancel all the even distortion products, polyphase multipath circuits with more than two paths will cancel even more distortion products.

Of course, just as for balanced circuits, the canceling depends on the matching between the paths. Also, just as for balanced circuits, the signal to noise ratio is not detoriated by the multipath technique. 


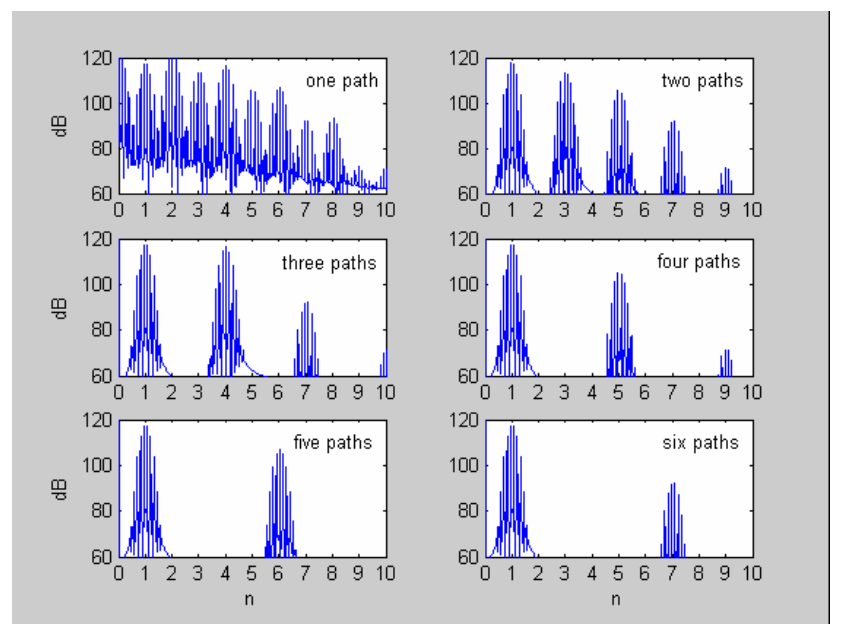

Figure 4: Simulation results for a polyphase multipath circuit (see figure 1).

Gingell [2] proposes to cancel the image signal of a mixer with the help of a polyphase filter before the mixer. He also makes use of multiple paths. Figure 3 shows the system he uses. He uses the polyphase filter to perform the first phase shifts. However, in his work there are no nonlinear circuits, so no harmonics of the input signal are produced.

We can compare his work with table 3 if we look at the columns with $n=-1$ and $n=1$. The image signal $(n=-1, j=1)$ has a phase of $2 \varphi$ and will be canceled by 3 or more paths (it can also be canceled by two paths, but then $\varphi$ should be $\left[0^{\circ}, 90^{\circ}\right]$ instead of $\left.\left[0^{\circ}, 180^{\circ}\right]\right)$. Gingell states that not only the image signal, but also the other spectral components up to and including the $(\mathrm{N}-2)^{\text {th }}$ harmonic of the LO are canceled with $\mathrm{N}$ paths. This can also be seen in table 3, looking at the column with $\mathrm{n}=-1$. For example, according to Gingell the first component that is not canceled for four paths will be the third harmonic of the LO. Table 3 gives a phase for this harmonic of $4 \varphi$. Table 2 shows that this is indeed the first phase that is not canceled with four paths. Table 3 , however, does not only show the effect of a polyphase multipath circuit on the harmonics of the LO signal, but also on the harmonics of the input signal, produced by nonlinear circuits.

\section{SIMULATIONS}

In order to check the theory of section two, some simulations were done. (Ideal) phase shifters and nonlinear circuits were implemented in Matlab and circuits with one to six paths as in figure 1 were built. The phases of the phase shifters were chosen equidistant.

Figure 4 shows the result of a two-tone test. The fourier transform (fft) of the output of the circuits was calculated. On the horizontal axis is the number of the harmonics, $n$. On the $y$ axis is the amplitude of the frequency components in $\mathrm{dB}$. The figure shows that the more paths are used, the more harmonics and intermodulation products are canceled. For $\mathrm{N}$ paths, the $\left(\mathrm{p}^{*} \mathrm{~N}+1\right)^{\text {th }}$ harmonics are the only components that are not canceled. The figure also shows that the intermodulation products around the desired frequency cannot be canceled. This result was predicted in section 2 .

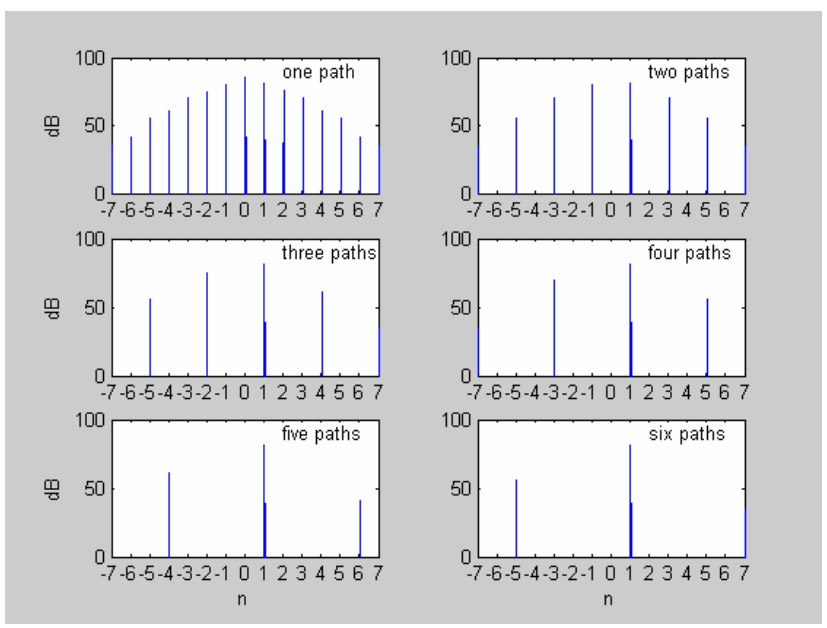

Figure 5: Simulation results for a polyphase multipath circuit with mixer (see figure 2).

Figure 5 shows a one-tone test of a circuit with mixers. In order to keep the figure clear, only the harmonics of the input signal around the first harmonic of the LO signal are plotted $(\mathrm{n}=$ 0 is at a frequency $\left.\omega_{\mathrm{LO}}\right)$. The simulations confirm that if a mixer is used instead of a phase shifter, the spectral components with $n$ $=\mathrm{j}+\mathrm{p} * \mathrm{~N}$ cannot be canceled by $\mathrm{N}$ paths. All the other spectral component can be canceled.

\section{CONCLUSIONS}

It has been shown that with polyphase multipath circuits it is very well possible to cancel distortion products produced by a nonlinear circuit. The more paths and phases are used, the more distortion products are canceled. In this paper tables are presented that can be used to determine whether a certain spectral component will be canceled or not depending on the number of paths. Unfortunately, some intermodulation products (like third order intermodulation products at $2 \omega_{1}-\omega_{2}$ and $2 \omega_{2}-$ $\omega_{1}$ ) cannot be canceled.

If a mixer is used as a phase shifter, extra spectral components appear: components in both an upper and a lower sideband around the harmonics of the local oscillator signal. Most of the components can be canceled by the polyphase multipath circuits if a sufficient number of paths is used. Thus an upconverter mixer can be made with a "clean" output spectrum.

\section{REFERENCES}

[1] W. Sansen, "Distortion in elementary transistor circuits," IEEE Transactions on Circuits and Systems II: Analog and Digital Signal Processing, Vol. 46, no. 3, pp. 315 - 325, March 1999.

[2] M. J. Gingell, "Single Sideband Modulation using Sequence Asymmetric Polyphase Networks," Elect. Commun., vol. 48, pp. $21-25,1973$.

[3] D.K. Weaver, "A Third Method of Generation and Detection of Single-Sideband Signals," Institute of Radio Engineers Proceedings, vol. 44, pp. 1703 - 1705, Dec 1956. 